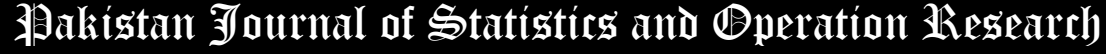

\section{Relations for Moments of Generalized Order Statistics for Transmuted Exponential Distribution and Characterization}

Mashail Al-Sobhi ${ }^{1}$, Saman Hanif Shahbaz, ${ }^{2, *}$ and Rehan Ahmad Khan Sherwani ${ }^{3}$

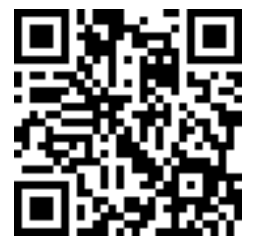

* Corresponding Author

1. Department of Mathematics, Umm Al-Qura University, Makkah, Saudi Arabia, mmsobhi@uqu.edu.sa

2. Department of Statistics, King Abdulaziz University, Jeddah, Saudi Arabia, shmohamad2@kau.edu.sa

3. College of Statistical and Actuarial Sciences, University of the Punjab, Pakistan, rehan.stat@pu.edu.pk

\begin{abstract}
The relations for moments of generalized order statistics ( $g o s)$ for transmuted exponential distribution are obtained. These include relations for single, inverse, product and ratio moments. These relations are useful in for recursive computation of moments of gos for transmuted exponential distribution. Some characterizations of the distribution, based on single and product moments of gos, are also obtained.
\end{abstract}

Key Words: Generalized Order Statistics, Transmuted Exponential Distribution, Recurrence Relations, Characterizations.

\section{Mathematical Subject Classification: 62G30, 62E10, 60E05}

\section{Introduction}

Generalized order statistics (gos) has been introduced by Kamps (1995) as a unified model for ordered random variables. Several models of ordered data including ordinary order statistics and record values, introduced by Chandler (1952), appears as a special case of gos. The joint density function of $n$ gos, introduced by Kamps (1995) is

$$
f_{1, \ldots, n: n, m, k}\left(x_{1}, x_{2}, \ldots, x_{n}\right)=k\left(\prod_{j=1}^{n-1} \gamma_{j}\right)\left\{1-F\left(x_{n}\right)\right\}^{k-1} f\left(x_{n}\right)\left[\prod_{j=1}^{n-1}\left\{1-F\left(x_{i}\right)^{m}\right\} f\left(x_{i}\right)\right],
$$

where $n, m$ and $k$ are parameters of the model. The quantities $\gamma_{j}$ are defined as $\gamma_{j}=k+(n-j)(m+1)$. The density function of a single gos, obtained by Kamps (1995), is

$$
f_{r: n, m, k}(x)=\frac{C_{r-1}}{(r-1) !} f(x)\{1-F(x)\}^{\gamma_{r}-1} g_{m}^{r-1}[F(x)]
$$

where $C_{r-1}=\prod_{j=1}^{r} \gamma_{j}$ and

$$
g_{m}(x)=h_{m}(x)-h_{m}(0)= \begin{cases}{\left[1-(1-x)^{m+1}\right] /(m+1) ;} & m \neq-1 \\ -\ln (1-x) & m=-1 .\end{cases}
$$

The joint density function of two gos is

$$
\begin{aligned}
f_{r, s: n, m, k}\left(x_{1}, x_{2}\right)= & \frac{C_{s-1}}{(r-1) !(s-r-1) !} f\left(x_{1}\right) f\left(x_{2}\right)\left\{1-F\left(x_{1}\right)\right\}^{m} g_{m}^{r-1}\left\{F\left(x_{1}\right)\right\} \\
& \times\left\{1-F\left(x_{2}\right)\right\}^{\gamma_{s}-1}\left[h_{m}\left\{F\left(x_{2}\right)\right\}-h_{m}\left\{F\left(x_{1}\right)\right\}\right]^{s-r-1} ;-\infty<x_{1}<x_{2}<\infty .
\end{aligned}
$$

where 


$$
h_{m}(x)= \begin{cases}-(1-x)^{m+1} /(m+1) ; & m \neq-1 \\ -\ln (1-x) & m=-1 .\end{cases}
$$

The gos is a unified model for the ordered random variables and different choices of the parameters provide different models of ordered data. For example the ordinary order statistics appears as a special case of $g o s$ for $m=0$ and $k=1$. The $k$ th record values, introduced by Dziubdziel and Kopociski (1976), appear as a special case of gos for $m=-1$. Also for $m=-1$ and $k=1$, the gos reduces to upper record values, introduced by Chandler (1952). A detailed discussion on gos can be found in Ahsanullah and Nevzorov (2001) and in Shahbaz et al. (2016).

Since its emergence, the gos has attracted several authors to study its properties for specific distributions. Most of the work in gos has been related with the recursive computations for moments of gos for a specific base distribution and characterizations based upon the moment of gos. Joshi (1978) has obtained relations for moments of ordinary order statistics for exponential distribution. Cramer and Kamps (2000) and Athar and Islam (2004) have provided general framework for relations between moments of gos for any base distribution. Kumar (2010) and Kumar (2011) has obtained relations for moments of gos for $p$ th order exponential distribution and Kumaraswamy distribution respectively. Khan et al. (2010) has obtained moments of gos for Erlang-truncated exponential distribution. Hanif Shahbaz and Shahbaz (2017) have obtained recurrence relations for moments of gos for Kumaraswamy Pareto distribution. Some other notable references include Ahsanullah et al. (2015), Balakrishnan et al. (2015), Kumar et al. (2019) among others.

The recurrence relations for moments of transmuted distributions have not been explored and in this paper we have obtained the recurrence relations for moments of gos for transmuted exponential distribution. We will first give a brief about the transmuted exponential distribution.

\section{Transmuted Exponential Distribution}

The exponential distribution has been a popular probability distribution in reliability and life testing. The density and distribution function of exponential distribution are

$$
f(x ; \alpha)=\alpha \exp (-\alpha x) \text { and } F(x)=1-\exp (-\alpha x) ; \alpha, x>0 .
$$

The distribution has been extensively studied and several generalizations of this distribution are proposed in literature. Owoloko et al. (2015) have used the quadratic transmutation technique, introduced by Shaw and Buckley (2007) to propose the transmuted exponential distribution. The density and distribution functions of the transmuted exponential distribution are, respectively, given as

$$
f(x)=\alpha e^{-\alpha x}\left[1+\lambda-2 \lambda\left(1-\mathrm{e}^{-\alpha x}\right)\right] ; x, \alpha>0,-1<\lambda<1
$$

and

$$
F(x)=(1+\lambda)\left(1-\mathrm{e}^{-\alpha x}\right)-\lambda\left(1-\mathrm{e}^{-\alpha x}\right)^{2} ; x, \alpha>0,-1<\lambda<1 .
$$

Al-Sobhi et al. (2020) has shown that the density and distribution function of the transmuted exponential distribution are related as

$$
[1-F(x)]=\frac{1}{\alpha} f(x)-\lambda \sum_{j=0}^{\infty}(-2)^{j} \frac{\alpha^{j} x^{j}}{j !} .
$$

Al-Sobhi et al. (2020) have used the relation (6) to obtain the recurrence relations for moments of ordinary order statistics for transmuted exponential distribution. In this paper we have presented the recurrence relations for moments of gos for the transmuted exponential distribution. We have also given some characterizations for transmuted exponential distribution based upon the moments of gos. These relations and characterizations are given in the following sections.

\section{Relations for Raw and Inverse Moments}

In this section we have presented the recurrence relations for single and inverse moments of gos for transmuted exponential distribution. These relations are given in the following Theorem and corollaries.

Theorem 1: The raw moments of gos for transmuted exponential distribution are related as

$$
\mu_{r: n, m, k}^{p}=\mu_{r-1: n, m, k}^{p}+\frac{p}{\alpha \gamma_{r}} \mu_{r: n, m, k}^{p-1}-\lambda \sum_{j=0}^{\infty}(-2)^{j} \frac{\alpha^{j} \gamma_{r(k-1)} C_{r-1}}{j ! \gamma_{r} C_{r-1(k-1)}}\left[\mu_{r: n, m, k-1}^{p+j}-\mu_{r-1: n, m, k-1}^{p+j}\right],
$$


where $\gamma_{j(k-1)}=(k-1)+(n-j)(m+1)$ and $C_{r-1(k-1)}=\prod_{j=1}^{r} \gamma_{j(k-1)}$.

Proof: Athar and Islam (2004) have shown that the moments of gos for any parent distribution are related as

$$
\mu_{r: n, m, k}^{p}-\mu_{r-1: n, m, k}^{p}=\frac{p C_{r-1}}{\gamma_{r}(r-1) !} \int_{-\infty}^{\infty} x^{p-1}\{1-F(x)\}^{\gamma_{r}} g_{m}^{r-1}[F(x)] d x
$$

where $\mu_{r: n, m, k}^{p}=E\left(X_{r: n, m, k}^{p}\right)$ and $X_{r: n, m, k}$ is $r$ th gos. The relation (8) can be written as

$$
\mu_{r: n, m, k}^{p}-\mu_{r-1: n, m, k}^{p}=\frac{p C_{r-1}}{\gamma_{r}(r-1) !} \int_{-\infty}^{\infty} x^{p-1}\{1-F(x)\}\{1-F(x)\}^{\gamma_{r}-1} g_{m}^{r-1}[F(x)] d x .
$$

Now using (6) in above equation we have

$$
\begin{aligned}
\mu_{r: n, m, k}^{p}-\mu_{r-1: n, m, k}^{p}= & \frac{p C_{r-1}}{\gamma_{r}(r-1) !} \int_{0}^{\infty} x^{p-1}\left[\frac{1}{\alpha} f(x)-\lambda \sum_{j=0}^{\infty}(-2)^{j} \frac{\alpha^{j} x^{j}}{j !}\right][1-F(x)]^{\gamma_{r}-1} g_{m}^{r-1}[F(x)] d x \\
& =\frac{p C_{r-1}}{\alpha \gamma_{r}(r-1) !} \int_{0}^{\infty} x^{p-1} f(x)[1-F(x)]^{\gamma_{r}-1} g_{m}^{r-1}[F(x)] d x \\
& -\lambda \sum_{j=0}^{\infty}(-2)^{j} \frac{\alpha^{j}}{j !} \frac{p C_{r-1}}{\gamma_{r}(r-1) !} \int_{0}^{\infty} x^{p+j-1}[1-F(x)]^{\gamma_{r}-1} g_{m}^{r-1}[F(x)] d x
\end{aligned}
$$

or

$$
\begin{aligned}
\mu_{r: n, m, k}^{p}-\mu_{r-1: n, m, k}^{p}= & \frac{p}{\alpha \gamma_{r}} \mu_{r: n, m, k}^{p-1}-\lambda \sum_{j=0}^{\infty}(-2)^{j} \frac{\alpha^{j}}{j !} \frac{p \gamma_{r(k-1)} C_{r-1} C_{r-1(k-1)}}{\gamma_{r(k-1)} \gamma_{r} C_{r-1(k-1)}(r-1) !} \int_{0}^{\infty} x^{p+j-1} \\
\times & {[1-F(x)]^{\gamma_{r}(k-1)} g_{m}^{r-1}[F(x)] d x } \\
= & \frac{p}{\alpha \gamma_{r}} \mu_{r: n, m, k}^{p-1}-\lambda \sum_{j=0}^{\infty}(-2)^{j} \frac{\alpha^{j} \gamma_{r(k-1)} C_{r-1}}{j ! \gamma_{r} C_{r-1(k-1)}} \frac{p C_{r-1(k-1)}}{\gamma_{r(k-1)}(r-1) !} \int_{0}^{\infty} x^{p+j-1} \\
\times & {[1-F(x)]^{\gamma_{r(k-1)}} g_{m}^{r-1}[F(x)] d x, }
\end{aligned}
$$

where $\gamma_{r(k-1)}=(k-1)+(n-r)(m+1)$ and $C_{r-1(k-1)}=\prod_{j=1}^{r} \gamma_{j(k-1)}$. Now using (6) again we have

or

$$
\begin{aligned}
& \mu_{r: n, m, k}^{p}-\mu_{r-1: n, m, k}^{p}=\frac{p}{\alpha \gamma_{r}} \mu_{r: n, m, k}^{p-1}-\lambda \sum_{j=0}^{\infty}(-2)^{j} \frac{\alpha^{j} \gamma_{r(k-1)} C_{r-1}}{j ! \gamma_{r} C_{r-1(k-1)}}\left[\mu_{r: n, m, k-1}^{p+j}-\mu_{r-1: n, m, k-1}^{p+j}\right] \\
& \mu_{r: n, m, k}^{p}=\mu_{r-1: n, m, k}^{p}+\frac{p}{\alpha \gamma_{r}} \mu_{r: n, m, k}^{p-1}-\lambda \sum_{j=0}^{\infty}(-2)^{j} \frac{\alpha^{j} \gamma_{r(k-1)} C_{r-1}}{j ! \gamma_{r} C_{r-1(k-1)}}\left[\mu_{r: n, m, k-1}^{p+j}-\mu_{r-1: n, m, k-1}^{p+j}\right],
\end{aligned}
$$

which is (5) and hence the result.

It is interesting to note that the recurrence relation (7) reduces to the recurrence relations for moments of gos from exponential distribution, obtained by Ahsanullah (2000), for $\lambda=0$. Also for $m=0$ and $k=1$, the relation (7) reduces to the relations for single moments of ordinary order statistics, obtained by Al-Sobhi (2020).

Some corollaries from Theorem 1 are given below.

Corollary 1: Replacing $p$ with $-p$ in (7), the recurrence relations for inverse moments of gos for transmuted exponential distribution is given as

$$
\mu_{r: n, m, k}^{-p}=\mu_{r-1: n, m, k}^{-p}-\frac{p}{\alpha \gamma_{r}} \mu_{r: n, m, k}^{-(p+1)}+\lambda \sum_{j=0}^{\infty}(-2)^{j} \frac{\alpha^{j} \gamma_{r(k-1)} C_{r-1}}{j ! \gamma_{r} C_{r-1(k-1)}}\left[\mu_{r: n, m, k-1}^{-p+j}-\mu_{r-1: n, m, k-1}^{-p+j}\right] .
$$

Corollary 2: Using $m=-1$ in (7) we have following recurrence relation for moments of record values for transmuted exponential distribution 


$$
\mu_{K(r)}^{p}=\mu_{K(r-1)}^{p}+\frac{p}{\alpha k} \mu_{K(r)}^{p-1}-\lambda \sum_{j=0}^{\infty} \frac{(-2)^{j} \alpha^{j}}{j !}\left(\frac{k}{k-1}\right)^{r-1}\left[\mu_{K-1(r)}^{p+j}-\mu_{K-1(r-1)}^{p+j}\right]
$$

Corollary 3: Using $m=-1$ in (9) we have following recurrence relation for inverse moments of record values for transmuted exponential distribution

$$
\mu_{K(r)}^{-p}=\mu_{K(r-1)}^{-p}-\frac{p}{\alpha k} \mu_{K(r)}^{-(p+1)}+\lambda \sum_{j=0}^{\infty} \frac{(-2)^{j} \alpha^{j}}{j !}\left(\frac{k}{k-1}\right)^{r-1}\left[\mu_{K-1(r)}^{-p+j}-\mu_{K-1(r-1)}^{-p+j}\right] .
$$

In the following section we will give the recurrence relations for product and ratio moments of gos for transmuted exponential distribution.

\section{Relations for Product and Ratio Moments}

In this section we have derived the recurrence relations for product and ratio moments of gos for transmuted exponential distribution. These relations are given in the following Theorem and corollaries.

Theorem 2: The product moments of gos for transmuted exponential distribution are related as

$$
\mu_{r, s: n, m, k}^{p, q}=\mu_{r, s-1: n, m, k}^{p, q}+\frac{q}{\alpha \gamma_{s}} \mu_{r, s: n, m, k}^{p, q-1}-\lambda \sum_{j=0}^{\infty}(-2)^{j} \frac{\alpha^{j} \gamma_{s(k-1)} C_{s-1}}{j ! \gamma_{s} C_{s-1(k-1)}}\left[\mu_{r, s, n, m, k-1}^{p, q}-\mu_{r, s-1: n, m, k-1}^{p, q}\right] .
$$

Proof: Athar and Islam (2004) have shown that the product moments of gos for any parent distribution are related as

$$
\begin{aligned}
\mu_{r, s, n, m, k}^{p, q}-\mu_{r, s-1: n, m, k}^{p, q} & =\frac{q C_{s-1}}{\gamma_{s}(r-1) !(s-r-1) !} \int_{-\infty}^{\infty} \int_{x_{1}}^{\infty} x_{1}^{p} x_{2}^{q-1} f\left(x_{1}\right)\left[1-F\left(x_{1}\right)\right]^{m} g_{m}^{r-1}\left[F\left(x_{1}\right)\right] \\
\times & {\left[h_{m}\left\{F\left(x_{2}\right)\right\}-h_{m}\left\{F\left(x_{1}\right)\right\}\right]^{s-r-1}\left[1-F\left(x_{2}\right)\right]^{\gamma_{s}} d x_{2} d x_{1}, }
\end{aligned}
$$

where $\mu_{r, s: n, m, k}^{p, q}=E\left(X_{r: n, m, k}^{p} X_{s: n, m, k}^{q}\right)$. The relation (13) can be written as

$$
\begin{aligned}
\mu_{r, s: n, m, k}^{p, q}-\mu_{r, s-1: n, m, k}^{p, q}= & \frac{q C_{s-1}}{(r-1) !(s-r-1) !} \int_{-\infty}^{\infty} \int_{x_{1}}^{\infty} x_{1}^{p} x_{2}^{q-1} f\left(x_{1}\right)\left[1-F\left(x_{1}\right)\right]^{m} g_{m}^{r-1}\left[F\left(x_{1}\right)\right] \\
\times & {\left[h_{m}\left\{F\left(x_{2}\right)\right\}-h_{m}\left\{F\left(x_{1}\right)\right\}\right]^{s-r-1}\left[1-F\left(x_{2}\right)\right]\left[1-F\left(x_{2}\right)\right]^{\gamma_{s}-1} d x_{2} d x_{1} . }
\end{aligned}
$$

Now, using (6) in above equation we have

$$
\begin{aligned}
& \mu_{r, s: n, m, k}^{p, q}-\mu_{r, s-1: n, m, k}^{p, q}=\frac{q C_{s-1}}{(r-1) !(s-r-1) !} \int_{-\infty}^{\infty} \int_{x_{1}}^{\infty} x_{1}^{p} x_{2}^{q-1} f\left(x_{1}\right)\left[1-F\left(x_{1}\right)\right]^{m} \\
& \times g_{m}^{r-1}\left[F\left(x_{1}\right)\right]\left[h_{m}\left\{F\left(x_{2}\right)\right\}-h_{m}\left\{F\left(x_{1}\right)\right\}\right]^{s-r-1} \\
& \times\left[\frac{1}{\alpha} f\left(x_{2}\right)-\lambda \sum_{j=0}^{\infty}(-2)^{j} \frac{\alpha^{j} x_{2}^{j}}{j !}\right]\left[1-F\left(x_{2}\right)\right]^{\gamma_{s}-1} d x_{2} d x_{1}
\end{aligned}
$$

or

$$
\begin{aligned}
\mu_{r, s: n, m, k}^{p, q}-\mu_{r, s-1: n, m, k}^{p, q} & =\frac{q C_{s-1}}{\alpha \gamma_{s}(r-1) !(s-r-1) !} \int_{-\infty}^{\infty} \int_{x_{1}}^{\infty} x_{1}^{p} x_{2}^{q-1} f\left(x_{1}\right) f\left(x_{2}\right)\left[1-F\left(x_{1}\right)\right]^{m} \\
& \times g_{m}^{r-1}\left[F\left(x_{1}\right)\right]\left[h_{m}\left\{F\left(x_{2}\right)\right\}-h_{m}\left\{F\left(x_{1}\right)\right\}\right]^{s-r-1}\left[1-F\left(x_{2}\right)\right]^{\gamma_{s}-1} d x_{2} d x_{1} \\
& -\lambda \sum_{j=0}^{\infty}(-2)^{j} \frac{\alpha^{j}}{j !} \frac{q C_{s-1}}{\gamma_{s}(r-1) !(s-r-1) !} \int_{-\infty}^{\infty} \int_{x_{1}}^{\infty} x_{1}^{p} x_{2}^{q+j-1} f\left(x_{1}\right)\left[1-F\left(x_{1}\right)\right]^{m} \\
& \times g_{m}^{r-1}\left[F\left(x_{1}\right)\right]\left[h_{m}\left\{F\left(x_{2}\right)\right\}-h_{m}\left\{F\left(x_{1}\right)\right\}\right]^{s-r-1}\left[1-F\left(x_{2}\right)\right]^{\gamma_{s}-1} d x_{2} d x_{1}
\end{aligned}
$$

or 


$$
\begin{aligned}
\mu_{r, s: n, m, k}^{p, q}-\mu_{r, s-1: n, m, k}^{p, q}=\frac{q}{\alpha \gamma_{s}} & \mu_{r, s: n, m, k}^{p, q-1}-\lambda \sum_{j=0}^{\infty}(-2)^{j} \frac{\alpha^{j}}{j !} \frac{q C_{s-1}}{\gamma_{s}(r-1) !(s-r-1) !} \\
\times & \int_{-\infty}^{\infty} \int_{x_{1}}^{\infty} x_{1}^{p} x_{2}^{q+j-1} f\left(x_{1}\right)\left[1-F\left(x_{1}\right)\right]^{m} g_{m}^{r-1}\left[F\left(x_{1}\right)\right] \\
\times & {\left[h_{m}\left\{F\left(x_{2}\right)\right\}-h_{m}\left\{F\left(x_{1}\right)\right\}\right]^{s-r-1}\left[1-F\left(x_{2}\right)\right]^{\gamma_{s}-1} d x_{2} d x_{1} }
\end{aligned}
$$

or

$$
\begin{aligned}
\mu_{r, s: n, m, k}^{p, q}-\mu_{r, s-1: n, m, k}^{p, q}=\frac{q}{\alpha \gamma_{s}} & \mu_{r, s: n, m, k}^{p, q-1}-\lambda \sum_{j=0}^{\infty}(-2)^{j} \frac{\alpha^{j}}{j !} \frac{q \gamma_{s(k-1)} C_{s-1} C_{s-1(k-1)}}{\gamma_{s} \gamma_{s(k-1)} C_{s-1(k-1)}(r-1) !(s-r-1) !} \\
\times & \int_{-\infty}^{\infty} \int_{x_{1}}^{\infty} x_{1}^{p} x_{2}^{q+j-1} f\left(x_{1}\right)\left[1-F\left(x_{1}\right)\right]^{m} g_{m}^{r-1}\left[F\left(x_{1}\right)\right] \\
\times & {\left[h_{m}\left\{F\left(x_{2}\right)\right\}-h_{m}\left\{F\left(x_{1}\right)\right\}\right]^{s-r-1}\left[1-F\left(x_{2}\right)\right]^{\gamma_{s(k-1)}} d x_{2} d x_{1} }
\end{aligned}
$$

where $\gamma_{s(k-1)}=(k-1)+(n-s)(m+1)$ and $C_{s-1(k-1)}=\prod_{j=1}^{s} \gamma_{j(k-1)}$. Now using (13) again we have

$$
\begin{aligned}
\mu_{r, s: n, m, k}^{p, q}-\mu_{r, s-1: n, m, k}^{p, q} & =\frac{q}{\alpha \gamma_{s}} \mu_{r, s: n, m, k}^{p, q-1}-\lambda \sum_{j=0}^{\infty}(-2)^{j} \frac{\alpha^{j} \gamma_{s(k-1)} C_{s-1}}{j ! \gamma_{s} C_{s-1(k-1)}}\left[\mu_{r, s, n, m, k-1}^{p, q}-\mu_{r, s-1: n, m, k-1}^{p, q}\right] \\
\text { or } \quad & \mu_{r, s: n, m, k}^{p, q}=\mu_{r, s-1: n, m, k}^{p, q}+\frac{q}{\alpha \gamma_{s}} \mu_{r, s, n, m, k}^{p, q-1}-\lambda \sum_{j=0}^{\infty}(-2)^{j} \frac{\alpha^{j} \gamma_{s(k-1)} C_{s-1}}{j ! \gamma_{s} C_{s-1(k-1)}}\left[\mu_{r, s: n, m, k-1}^{p, q}-\mu_{r, s-1: n, m, k-1}^{p, q}\right],
\end{aligned}
$$

which is (10) and hence the Theorem.

It is interesting to note that the recurrence relation (12) reduces to the recurrence relations for product moments of gos from exponential distribution, obtained by Ahsanullah (2000), for $\lambda=0$. Also for $m=0$ and $k=1$, the relation (12) reduces to the relations for single moments of ordinary order statistics, obtained by Al-Sobhi (2020).

Some corollaries from Theorem 2 are given below.

Corollary 4: Replacing $p$ with $-p$ in (12), the recurrence relations for ratio moments of gos for transmuted exponential distribution is given as

$$
\mu_{r, s: n, m, k}^{p,-q}=\mu_{r, s-1: n, m, k}^{p,-q}-\frac{q}{\alpha \gamma_{s}} \mu_{r, s: n, m, k}^{p,-(q+1)}+\lambda \sum_{j=0}^{\infty}(-2)^{j} \frac{\alpha^{j} \gamma_{s(k-1)} C_{s-1}}{j ! \gamma_{s} C_{s-1(k-1)}}\left[\mu_{r, s: n, m, k-1}^{p,-q}-\mu_{r, s-1: n, m, k-1}^{p,-q}\right] .
$$

Corollary 5: Using $m=-1$ in (12) we have following recurrence relation for product moments of record values for transmuted exponential distribution

$$
\mu_{K(r, s)}^{p, q}=\mu_{K(r, s-1)}^{p, q}+\frac{q}{\alpha k} \mu_{K(r, s)}^{p, q-1}-\lambda \sum_{j=0}^{\infty} \frac{(-2)^{j} \alpha^{j}}{j !}\left(\frac{k}{k-1}\right)^{r-1}\left[\mu_{K-1(r, s)}^{p, q}-\mu_{K-1(r, s-1)}^{p, q}\right] .
$$

Corollary 6: Using $m=-1$ in (15) we have following recurrence relation for ratio moments of record values for transmuted exponential distribution

$$
\mu_{K(r, s)}^{p,-q}=\mu_{K(r, s-1)}^{p,-q}-\frac{q}{\alpha k} \mu_{K(r, s)}^{p,-(q+1)}+\lambda \sum_{j=0}^{\infty} \frac{(-2)^{j} \alpha^{j}}{j !}\left(\frac{k}{k-1}\right)^{r-1}\left[\mu_{K-1(r, s)}^{p,-q}-\mu_{K-1(r, s-1)}^{p,-q}\right] .
$$

We will now give some characterizations of the transmuted exponential distribution in terms of moments of gos.

\section{Characterizations}

In this section we have given some characterizations of the transmuted exponential distribution in terms of moments of gos. These characterizations are given in the following theorems. 
Theorem 3: A necessary and sufficient condition for a random variable $X$ to have density and distribution function (3) and (4) respectively is that the moments of its gos are related as

$$
\mu_{r: n, m, k}^{p}-\mu_{r-1: n, m, k}^{p}=\frac{p}{\alpha \gamma_{r}} \mu_{r: n, m, k}^{p-1}-\lambda \sum_{j=0}^{\infty}(-2)^{j} \frac{\alpha^{j} \gamma_{r(k-1)} C_{r-1}}{j ! \gamma_{r} C_{r-1(k-1)}}\left[\mu_{r: n, m, k-1}^{p+j}-\mu_{r-1: n, m, k-1}^{p+j}\right] .
$$

Proof: The necessary condition immediately follow from Theorem 1. In order to prove the sufficient condition consider the relation (8) and hence we have

$$
\frac{p C_{r-1}}{\gamma_{r}(r-1) !} \int_{-\infty}^{\infty} x^{p-1}[\bar{F}(x)]^{\gamma_{r}} g_{m}^{r-1}[F(x)] d x=\frac{p}{\alpha \gamma_{r}} \mu_{r: n, m, k}^{p-1}-\lambda \sum_{j=0}^{\infty}(-2)^{j} \frac{\alpha^{j} \gamma_{r(k-1)} C_{r-1}}{j ! \gamma_{r} C_{r-1(k-1)}}\left[\mu_{r: n, m, k-1}^{p+j}-\mu_{r-1: n, m, k-1}^{p+j}\right]
$$

or

$$
\begin{aligned}
\frac{p}{\gamma_{r}} \frac{C_{r-1}}{(r-1) !} \int_{0}^{\infty} x^{p-1}[\bar{F}(x)]^{\gamma_{r}} g_{m}^{r-1}[F(x)] d x=\left[\frac{C_{r-1}}{(r-1) !} \int_{-\infty}^{\infty} x^{p-1}\{\bar{F}(x)\}^{\gamma_{r}-1} g_{m}^{r-1}\{F(x)\}\right. \\
\left.\times \frac{p}{\gamma_{r}}\left\{\frac{1}{\alpha} f(x)-\lambda \sum_{j=0}^{\infty}(-2)^{j} x^{p-1+j}\right\} d x\right]
\end{aligned}
$$

or

$$
\frac{p}{\gamma_{r}} \frac{C_{r-1}}{(r-1) !} \int_{0}^{\infty} x^{p-1}[\bar{F}(x)]^{\gamma_{r}-1} g_{m}^{r-1}[F(x)]\left[\bar{F}(x)-\left\{\frac{1}{\alpha} f(x)-\lambda \sum_{j=0}^{\infty}(-2)^{j} \frac{\alpha^{j} x^{j}}{j !}\right\}\right] d x=0
$$

Using Müntz-Száz theorem; see Hwang and Lin (1984); to above equation we have

$$
\bar{F}(x)=\left\{\frac{1}{\alpha} f(x)-\lambda \sum_{j=0}^{\infty}(-2)^{j} \frac{\alpha^{j} x^{j}}{j !}\right\},
$$

which is (6) and this relationship holds between density and distribution function of transmuted exponential distribution hence the theorem.

Theorem 4: A necessary and sufficient condition for a random variable $X$ to have density and distribution function (3) and (4) respectively is that the product moments of its gos are related as

$$
\mu_{r, s: n, m, k}^{p, q}-\mu_{r, s-1: n, m, k}^{p, q}=\frac{q}{\alpha \gamma_{s}} \mu_{r, s: n, m, k}^{p, q-1}-\lambda \sum_{j=0}^{\infty}(-2)^{j} \frac{\alpha^{j} \gamma_{s(k-1)} C_{s-1}}{j ! \gamma_{s} C_{s-1(k-1)}}\left[\mu_{r, s: n, m, k-1}^{p, q}-\mu_{r, s-1: n, m, k-1}^{p, q}\right] .
$$

Proof: The necessary part immediately follow from Theorem 2. For sufficient part we consider (13) as

$$
\begin{gathered}
\mu_{r, s: n, m, k}^{p, q}-\mu_{r, s-1: n, m, k}^{p, q}=\frac{q C_{s-1}}{\gamma_{s}(r-1) !(s-r-1) !} \int_{-\infty}^{\infty} \int_{x_{1}}^{\infty} x_{1}^{p} x_{2}^{q-1} f\left(x_{1}\right)\left[\bar{F}\left(x_{1}\right)\right]^{m} g_{m}^{r-1}[F(x)] \\
\times\left[h_{m}\left\{F\left(x_{2}\right)\right\}-h_{m}\left\{F\left(x_{1}\right)\right\}\right]^{s-r-1}\left[\bar{F}\left(x_{2}\right)\right]^{\gamma_{s}} d x_{2} d x_{1} .
\end{gathered}
$$

Now using above relation with (12) we have 


$$
\begin{aligned}
\frac{q C_{s-1}}{\gamma_{s}(r-1) !(s-r-1) !} \int_{0}^{\infty} \int_{x_{1}}^{\infty} x_{1}^{p} x_{2}^{q-1} f\left(x_{1}\right)\left[\bar{F}\left(x_{1}\right)\right]^{m} g_{m}^{r-1}\left[F\left(x_{1}\right)\right]\left[h_{m}\left\{F\left(x_{2}\right)\right\}-h_{m}\left\{F\left(x_{1}\right)\right\}\right]^{s-r-1} \\
\times\left[\bar{F}\left(x_{2}\right)\right]^{\gamma_{s}} d x_{2} d x_{1}=\left[\frac{C_{s-1}}{(r-1) !(s-r-1) !} \int_{0}^{\infty} \int_{x_{2}}^{\infty} x_{1}^{p} x_{2}^{q-1} f\left(x_{1}\right)\left\{\bar{F}\left(x_{1}\right)\right\}^{m} g_{m}^{r-1}\left\{F\left(x_{1}\right)\right\}\right. \\
\left.\quad \times\left\{h_{m}\left(F\left(x_{2}\right)\right)-h_{m}\left(F\left(x_{1}\right)\right)\right\}^{s-r-1}\{F(x)\}^{\gamma_{s}-1} \frac{p}{\gamma_{s}}\left\{\frac{1}{\alpha} f\left(x_{2}\right)-\lambda \sum_{j=0}^{\infty}(-2)^{j} \frac{\alpha^{j} x_{2}^{j}}{j !}\right\} d x_{2} d x_{1}\right]
\end{aligned}
$$

or

$$
\begin{gathered}
\frac{q C_{s-1}}{\gamma_{s}(r-1) !(s-r-1) !} \int_{0}^{\infty} \int_{x_{1}}^{\infty} x_{1}^{p} x_{2}^{q-1} f\left(x_{1}\right)\left[\bar{F}\left(x_{1}\right)\right]^{m} g_{m}^{r-1}\left[F\left(x_{1}\right)\right]\left[h_{m}\left\{F\left(x_{2}\right)\right\}-h_{m}\left\{F\left(x_{1}\right)\right\}\right]^{s-r-1} \\
\times\left[\bar{F}\left(x_{2}\right)\right]^{\gamma_{s}-1}\left[\bar{F}\left(x_{2}\right)-\left\{\frac{1}{\alpha} f\left(x_{2}\right)-\lambda \sum_{j=0}^{\infty}(-2)^{j} \frac{\alpha^{j} x_{2}^{j}}{j !}\right\}\right] d x_{2} d x_{1}=0
\end{gathered}
$$

Using Müntz-Száz theorem; see Hwang and Lin (1984); to above equation we have

$$
\bar{F}\left(x_{2}\right)=\left\{\frac{1}{\alpha} f\left(x_{2}\right)-\lambda \sum_{j=0}^{\infty}(-2)^{j} \frac{\alpha^{j} x_{2}^{j}}{j !}\right\},
$$

which is (6) and hence the theorem.

\section{Conclusions}

In this paper we have derived some relations for recursive computation of moments of gos for transmuted exponential distribution. These relations are useful in computing higher order moments from the corresponding lower order moments. These relations can also be used to obtain the recurrence relations for moments of special cases of gos. We have also obtained some characterizations for transmuted exponential distribution in terms of moments of gos.

\section{References}

Ahsanullah, M. (2000). Generalized order statistics from exponential distribution. Journal of Statistical Planning and Inference, 85, 85-91.

Ahsanullah, M, Hamedani, G.G and Maadooliat, M (2015). Characterizations of Distributions via Conditional Expectation of Generalized Order Statistics, International Journal of Statistics and Probability; 4( 3); 121-126. Ahsanullah, M. and Nevzorov, V. B. (2001). Ordered Random Variables, Nova Science Publishers, USA.

Al-Sobhi, M., Hanif Shahbaz, S. and Shahbaz, M. Q. (2020). Recursive Computation for Moments of Order Statistics for Transmuted Exponential Distribution, Advances and Applications in Statistics, 63, 175-189.

Athar, H. and Islam, H. M. (2004). Recurrence relations between single and product moments of generalized order statistics from a general class of distributions. Metron, LXII, 327-337.

Balakrishnan, N., Zhu, X. and Al-Zaharani, X. (2015). Recursive computation of the single and product moments of order statistics for the complementary exponential-geometric distribution. Journal of Statistical Computation and Simulation, 85, 2187-2201.

Chandler, K. N. (1952). The distribution and frequency of record values, Journal of the Royal Statistical Society, Series B, 14, 220-228.

Cramer, E. and Kamps, U., (2000). Relations for expectations of functions of generalized order statistics. Journal Statistical Planning and Inference, 89, 79-89.

Dziubdziel, W. A. and Kopociski, B. (1976). Limiting properties of the k-th record value. Applied Mathematics, 15,187-190

Hanif Shahbaz, S. and Shahbaz, M. Q. (2017). Recurrence Relations for Moments of Generalized Order Statistics from Kumaraswamy Pareto Distribution, Journal of Applied Statistical Sciences, 22, 429-442.

Kamps, U. (1995). A concept of generalized order statistics, Journal of Statistical Planning and Inference, 48, 123.

Hwang, J. S. and Lin, G. D., (1984). Extensions of Müntz-Száz theorems and application. Analysis, 4, 143-160. Joshi, P. C. (1978). Recurrence relations between moments of order statistics from exponential and truncated exponential distribution, Sankhya $B, 39,362-371$.

Khan, R.U., Kumar, D. and Ather, H., (2010). Moments of generalized order statistics from Erlang-Truncated exponential distribution and its characterization. International Journal Statistics and Systems, 5, 455-464. 
Kumar, D. (2010). Recurrence relations for single and product moments of generalized order statistics from pth order exponential distribution and its characterization. Journal of Statistical Research of Iran, 7, 101-112.

Kumar, D. (2011). Generalized Order Statistics from Kumaraswamy Distribution and its Characterization, Tamsui Oxford Journal of Information and Mathematical Sciences, 27, 463-476.

Kumar, D., Dey, S., Malik, M. R. and Shahbaz, M. Q. (2019). Recurrence Relations for Moments and Estimation of Parameters of Extended Exponential Distribution based on Progressive Type-II Right Censored Order Statistics, Journal of Statistical Theory and Applications, 18, 171-181.

Owoloko, E. A., Oguntunde, P. E. and Adejumo, A. O. (2015). Performance rating of the transmuted exponential distribution: An analytical approach. Springer Plus, 4, 8-18.

Shahbaz, M. Q., Ahsanullah, M., Hanif Shahbaz, S. and Al-Zahrani, B. (2016). Ordered Random Variables: Theory and Applications, Atlantis Studies in Probability and Statistics, Springer.

Shaw, W. T. and Buckley, I. R. C. (2007). The alchemy of probability distributions: beyond Gram-Charlier expansions, and a skew-kurtotic-normal distribution from a rank transmutation map. Research report. 